\title{
Data mining or data moaning?
}

Received: 22nd February, 2000

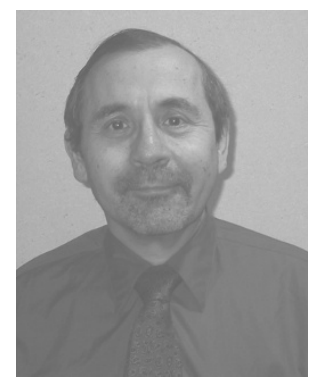

\section{Sam Koslowsky}

is currently Vice-President of Modeling Solutions at Harte Hanks Analytics. His responsibilities include providing strategic and quantitative solutions to database marketers. Sam spent eight years with American Express, the last four as Vice-President of Database Marketing, Modeling, and Analysis. Well experienced in CRM-related initiatives, Sam is a previous recipient of the coveted silver medal, awarded by the Direct Marketing Association for 'excellence in database marketing'.

$\mathrm{He}$ is a frequent speaker at industry meetings and publishes regularly in direct marketing-related journals. Sam also lectures at New York University in their direct marketing curriculum. He has an MBA in finance from New York University, where he was also granted an Advanced Professional Certificate for postgraduate study.

Abstract Perhaps no other single piece of technology has had more of an impact on database marketing than data mining. With powerful computers and polished software, marketers can convert data to information. Leveraging this asset provides a real competitive advantage. Many have been led to believe, however, that the technology itself, without user input, can result in significant benefits. While data mining should be part of an analyst's toolbox, by itself, the software can severely harm an otherwise well conceived marketing programme. The paper demonstrates the folly of letting data mining operate by itself.

Every so often, new technologies appear that claim to be the ultimate solution to information management challenges. Occasionally these assertions have some validity. Other times, however, these statements are somewhat exaggerated. Upon further investigation, it may be determined that it is the developers of these technologies that proclaim the power of these new advances. At a recent industry conference, a speaker announced that his firm would be releasing a vital piece of software that would eliminate 'the need for a trained individual to perform data mining'. At the same symposium, literature was distributed to the attendees that proclaimed that data mining would 'discern patterns in data that were impossible to detect with previous tools'.

What is most alarming about these developments is that many critical analytic related issues and other problems encountered during various stages of database marketing campaigns are frequently overlooked. Yet, for an accurate understanding of data, these issues simply cannot be ignored.

Data mining software should be found in most analysts' toolbox, and it clearly performs some essential functions. It does not, however, confront important issues such as inadequate data documentation, ambiguous project objectives, questionable file extracts, problematic sample designs and analyst inexperience. It is these, and similar concerns that, the author believes, have damaged the otherwise useful nature of data mining software.

\section{THE ROLE OF DATA MINING}

While a variety of definitions have been offered for 'data mining, most often it is characterised by automatic or, at the very 
minimum, semi-automatic, analysis of huge databases using a set of technologies including, but not limited to, rigorous statistical tools, tree-oriented investigations and neural network typologies. The primary purpose of all this is to discern patterns in data that would provide a benefit to marketers.

Ten years ago, the computer power necessary to make data mining a reality was not readily available. It was limited to a few select firms that were willing to invest in these approaches. With the efficiency of computer hardware and software improving significantly in the last decade, the use of analysis tools such as data mining is no longer a rarity. Indeed, it may be overused, and certainly in the wrong hands, results produced from a data mining effort may be meaningless.

The enthusiasm surrounding data mining peaked in the early 1990s. These 'new' techniques, proclaimed marketers and software developers alike, would be of significant value to database marketers. Expectations were high.

Reality set in in the middle of the decade, when marketers realised two things.

- the difficult-to-spot patterns that data mining were uncovering were basically identifiable already using existing techniques

- this 'new' software was not really new at all; rather, existing tools were brought together under an 'umbrella' package with an excellent graphical user interface.

Data mining essentially consists of four stages:

- definition of objective

- data preparation and exploratory data analysis

— data analysis
— post-data mining.

Definition of objective is an agreement among all parties as to what needs to be studied. This is not trivial, as will be demonstrated below.

Data preparation and exploratory data analysis focuses on three components:

- assembling data in a format that makes them amenable to analysis

- interrogating data to identify potential valuable predictors and descriptors

- transforming data to arrive at new, more meaningful predictors.

Data analysis is the part that is typically associated with data mining - number crunching and detection of patterns.

Post-data mining, the final stage, requires interpretation of results, quality control and subsequent evaluation.

While many marketers find it difficult to believe, the 'data preparation and data exploration' stage requires the most resources - at least 60 per cent of the time is typically devoted to this part of a successful data mining effort.

Several brief case histories that deal with each of the above four phases are now examined.

\section{THE DATA MINING OBJECTIVE}

\section{The insurance story}

A major financial services firm, in addition to providing standard offerings, also affords its customers the opportunity to register for an air flight insurance programme. The programme assesses a premium when an air ticket is charged to a credit card. In the unlikely event of a catastrophic air disaster, the insurer will pay the value of the policy. As accidents do not typically occur, the profits generated from this programme can be quite lucrative. 


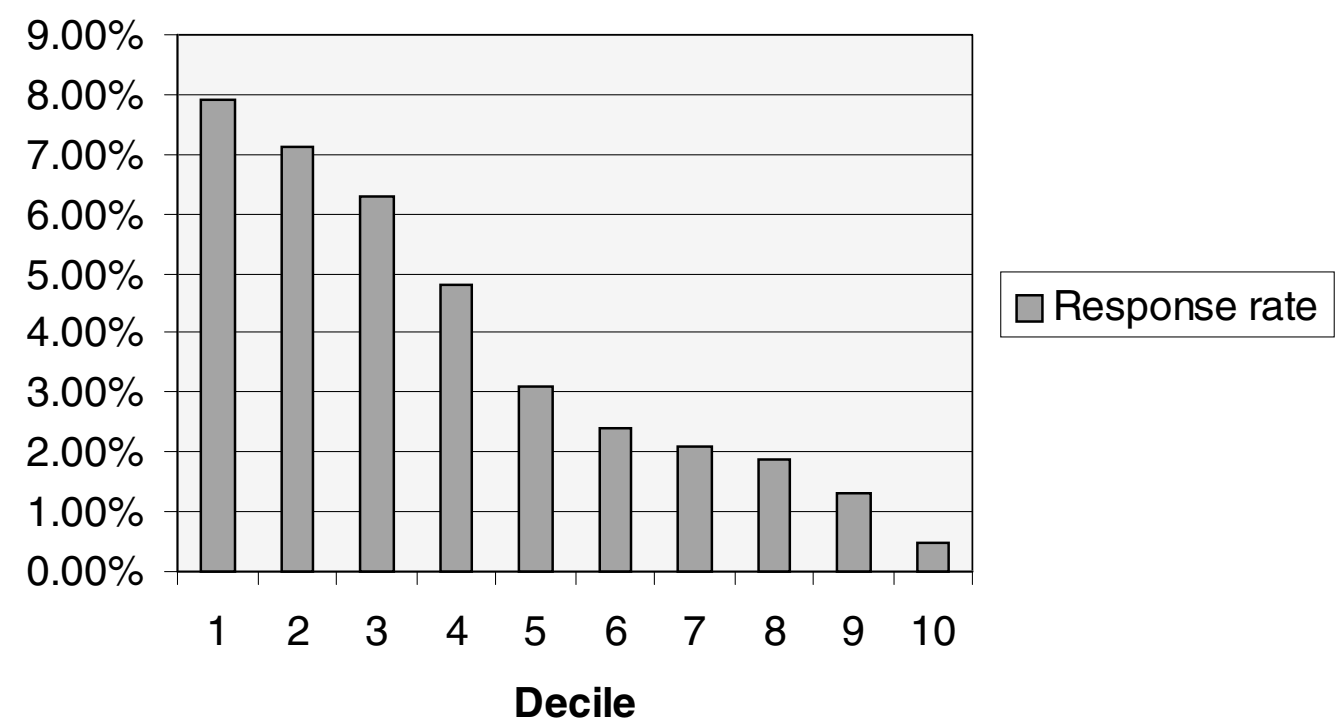

Figure 1 Air flight mailing

The firm commissioned a data mining study to determine who, among the company's customers, would be likely to register for this service. A model was developed, and results from the mailing were outstanding. Or were they?

The graph in Figure 1 displays the results by decile. A decile is no more than a way of grouping the population by likelihood to respond. Each decile represents 10 per cent of the population. Decile 1, as expected, was the best performing segment. Decile 10, the poorest. The analyst was pleased with the results. The marketer had mailed a significant number of names in the top deciles, anticipating correctly that the bulk of the respondents would be from these groups. The marketer mailed the bottom segments to make sure that the data mining effort that produced these results was working. By observing a lower response rate in the expected poorer groups, the marketer was confident that the model was performing well.

Everything seemed to be going smoothly. Six months after the mailing, the marketer decided to analyse where the revenue was coming from. The marketer determined that examining the revenue performance by decile would be appropriate. The results are shown in Figure 2.

It can be seen that the revenue was coming from the mid-range deciles, not the top segments, as expected. It appears that the most likely respondents to the direct mail offer were not the ones that were actually flying. And flying, not response, generates revenue.

No data mining software will be able to think through the correct objective for a project. In this case a dual model approach might have been more productive. One analysis would focus on response, the second on revenue.

An incorrect objective, or an incorrect assumption, can and will result in useless information.

\section{The cataloguer}

A cataloguer was expecting an exciting holiday season. Preparations for a mailing were being made. The data miner spent 


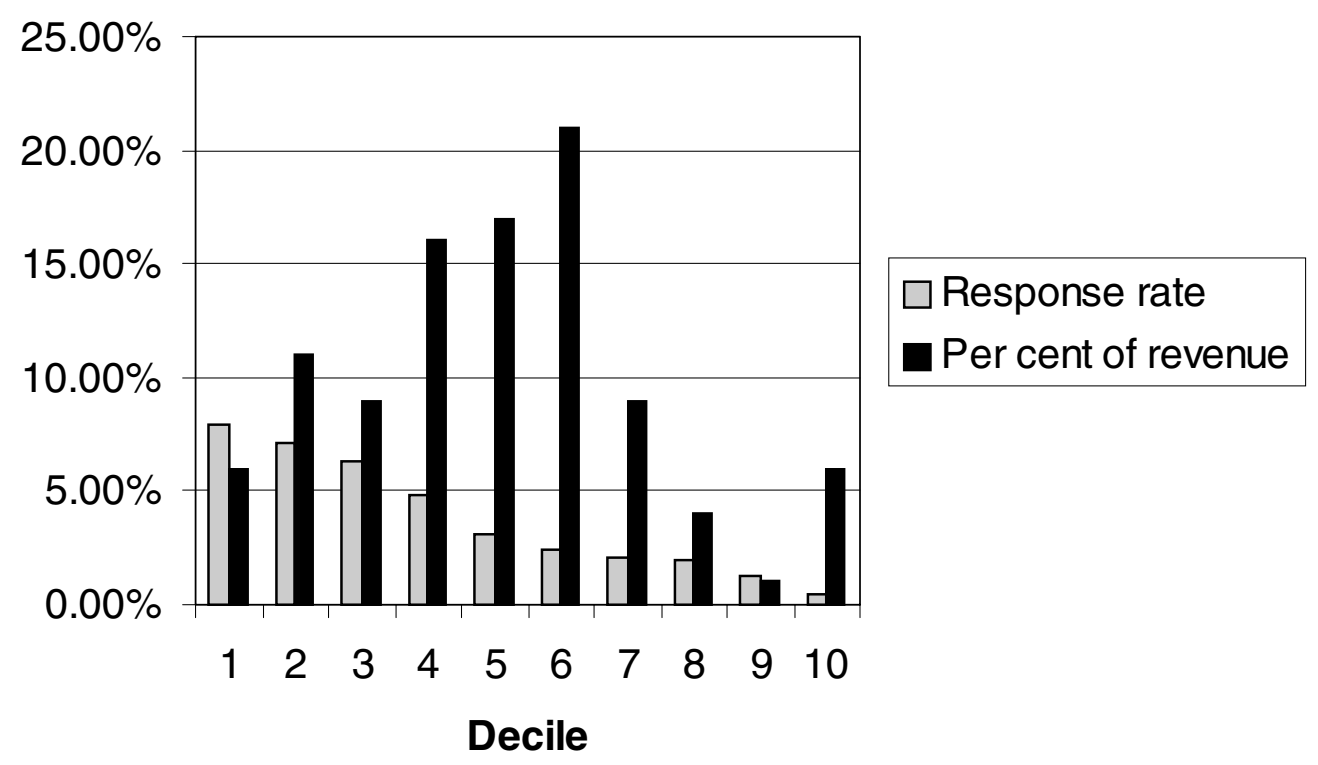

Figure 2 Response vs revenue

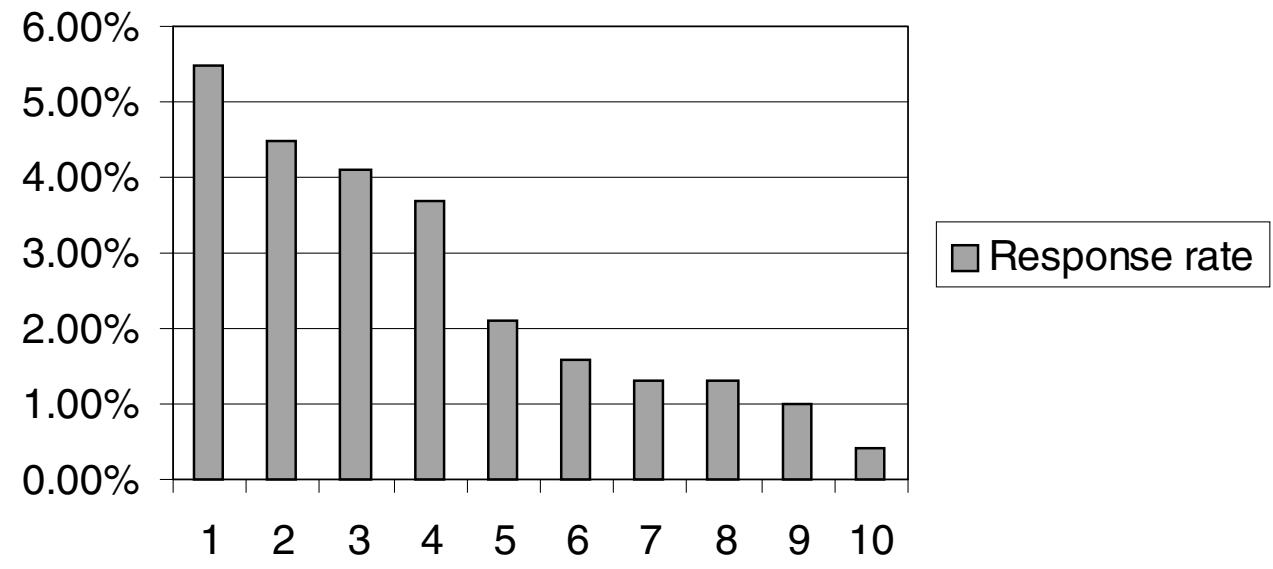

Figure 3 Cataloguer response rate

three weeks merging files to construct a model to predict response. Results were tabulated during the first week of February. The graph summarising the campaign is in Figure 3. One would anticipate finding such picture perfect results in a primer on data mining. The cataloguer, however, like similar firms in the industry, has a no-questions-asked return policy. Returns averaged 37.5 per cent of gross sales. The expected revenue was not materialising. In this case the more appropriate objective may have been modelling net revenue, not gross. Again, even the most powerful data mining software could not have detected this critical issue.

\section{DATA PREPARATION}

A critical early component of data mining is data preparation and 


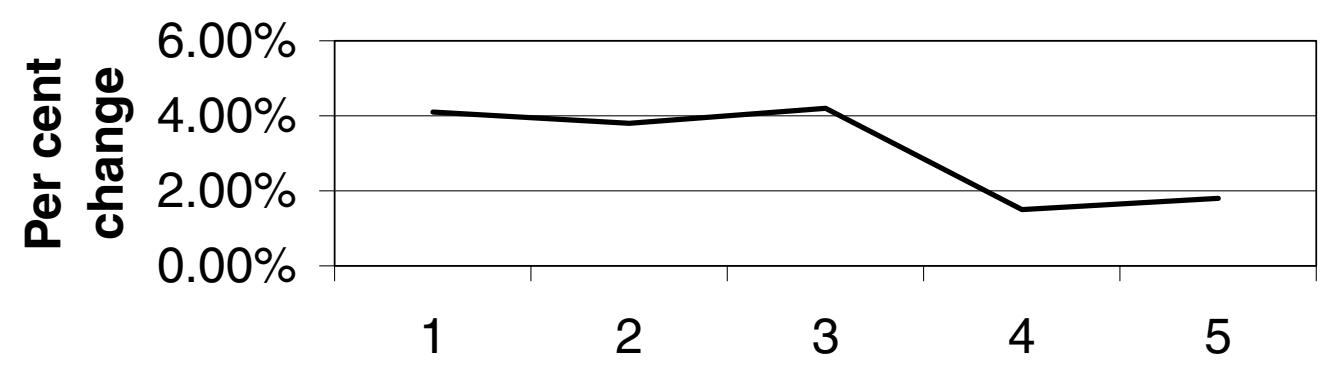

Period

Figure 4 Change in spending this year vs last year

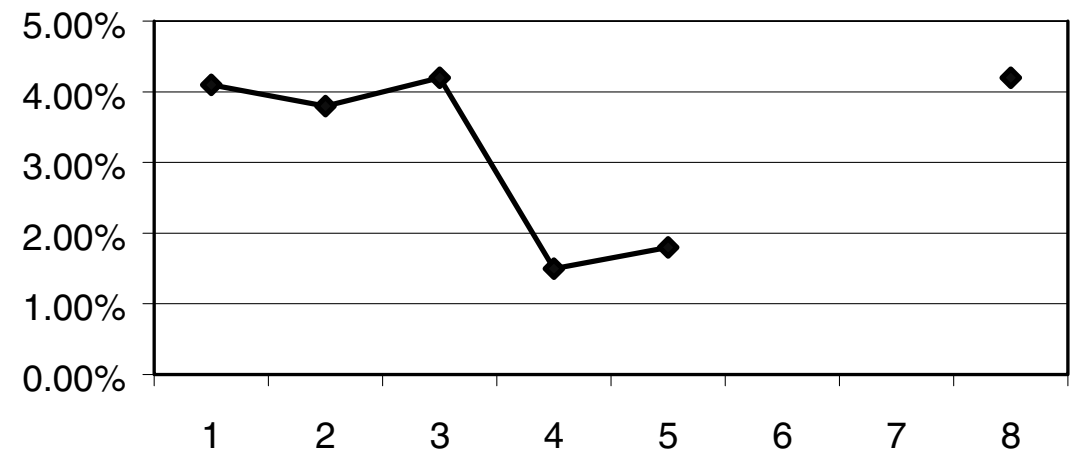

Figure 5

exploration, and the interpretation of the results. An example is a clothing retailer that had a fairly robust database. Sales were captured for all product lines, and were eventually summarised by customer at the department level. There were 14 such departments. Relative changes in spending during a 24-month period (in eight quarters) were noted. Results for the first five quarters appear in Figure 4.

There was a significant decrease in spending from Quarter 3 to Quarter 4. While this may very well be because of economic issues, the sudden change was puzzling. Management was not sure why this was happening, and were almost ready to attribute the downturn to economic factors.

This 'change' or velocity variable appeared in the final model. The model was now ready to be used.
Before model implementation, the phenomenon shown in Figure 5 was observed. Period 8 now showed an increase in line with patterns that were prevalent in the earlier periods.

The model took into account the change, especially the downward sloping curve. The trend in a subsequent period had now been reversed. In itself, this may not be bad. If the pattern change occurred naturally, then the data model built on this fluctuation would still be in fine shape. What, however, if the declining sales pattern was because of a data irregularity and not economics, then as the firm proceeds to roll out the model, results might be based on faulty input. And this sudden reversal to original trends would be all the more mysterious. Indeed, a further investigation was needed. 


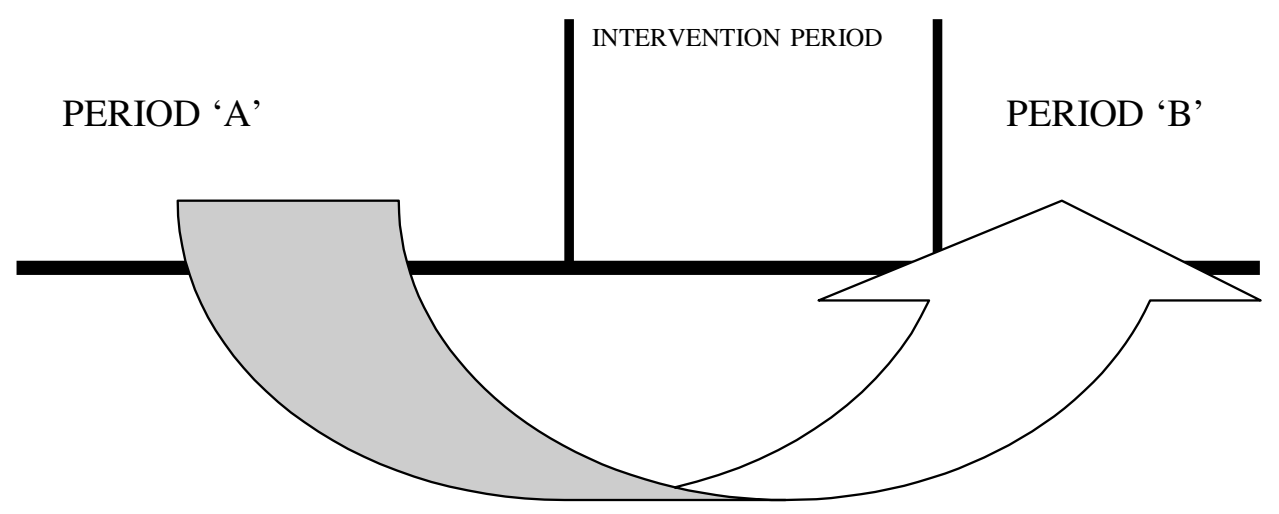

Figure 6

Further analysis and questioning showed that the merchandise department had removed three product lines from one department. This is why sales went down. The declining trend would only continue for one year because comparisons were being made with an earlier period when the department had more merchandise lines - like was not being compared with like. After 12 months (four quarters) comparisons would be 'fairer'.

It is highly unlikely that any data mining technology could locate the anomaly - never mind the change in merchandising being the reason for the anomaly. What is needed is a thinking, experienced analyst capable of questioning and exploring. Intuition, and professional knowledge of the business operation, help as well.

\section{Timing of data collection}

One of the key deliverables in a modelling exercise is the predictors. Predictors are necessary to predict future behaviour and to help explain or to describe what is happening. Frequently, these items are precursors for developing hypotheses concerning why, for example, customers are leaving or potential customers are responding.
While the output of a model should not be used for establishing cause and effect, many marketers continue to rely on models to arrive at cause-and-effect conclusions.

Selecting the correct predictors is essential for a successful marketing campaign. In the following example a telecommunications marketer was seeking to construct a retention programme. With the plethora of communications firms sprouting up, this company had developed a thorough and well-conceived strategy for retaining customers.

One of the steps involved targeting those customers who were likely to switch carriers. By determining early on a customer's probability of defecting, the firm could focus on these customers, and attempt to 'save' them. The programmes also incorporated a forecast of revenue by customer. By merging attrition likelihood, and future revenue generation by customer, the telecommunications giant would then be able to target its marketing resources to the appropriate individuals.

Typically, a defection model is constructed as follows. Predictors from, say Period ' $A$ ' are used to calculate the probability of a customer defecting in Period 'B'. Figure 6 may help clarify the 




Figure 7 Attrition rate

matter. Predictors are collected and analysed in period ' $A$ ' to calculate probability of switching in period 'B.' The interval in between provides the marketer with an opportunity to 'save' the customer.

A model was constructed using the above paradigm. And results, at least on paper, were outstanding. The decile performance report is shown in Figure 7. As can be seen, most of the defecting customers emanate from the first two deciles, a result that clearly pleased the marketer. By focusing appropriate resources where most of the problem lies, in these top segments, it was surmised a dent could be made in halting defection.

One of the author's cardinal beliefs is that if results look too good to be true, they are probably false. Further analysis indicated that the primary predictor was a data element that was referred to as 'service indicator'. This field almost always took on a value of ' 1 ' when subsequent defection occurred. The indicator was populated with a ' 0 ' in the absence of defection. The data mining software selected this variable to incorporate into this model. This seemed like a natural thing to do, according to the software logic. It was discovered, however, that when a customer defects, this field automatically converts to ' 1 ' during period 'B.' Essentially, this flag can be defined as someone defecting. Files were prepared for this analysis with this 'service indicator' appearing in Period 'A.' The marketer had used the fact that someone defected to predict that they would defect. Only an analyst capable of looking over the results, and inquiring into what was going on would be able to capture the folly of the above analysis. Data mining software could not, by itself, question, and consequently invalidate the results.

\section{DATA ANALYSIS}

An electronics cataloguer mined its file with the intention of producing a set of rules or models that would help in selecting future names for various marketing contacts. Data mining software was used and a result of the analysis showed that first-time buyers responded at a higher rate than multiple-time buyers. This conclusion is displayed in Figure 8 . The general rule in the catalogue industry is the reverse: that multiple-time purchasers will outperform 




Figure 8

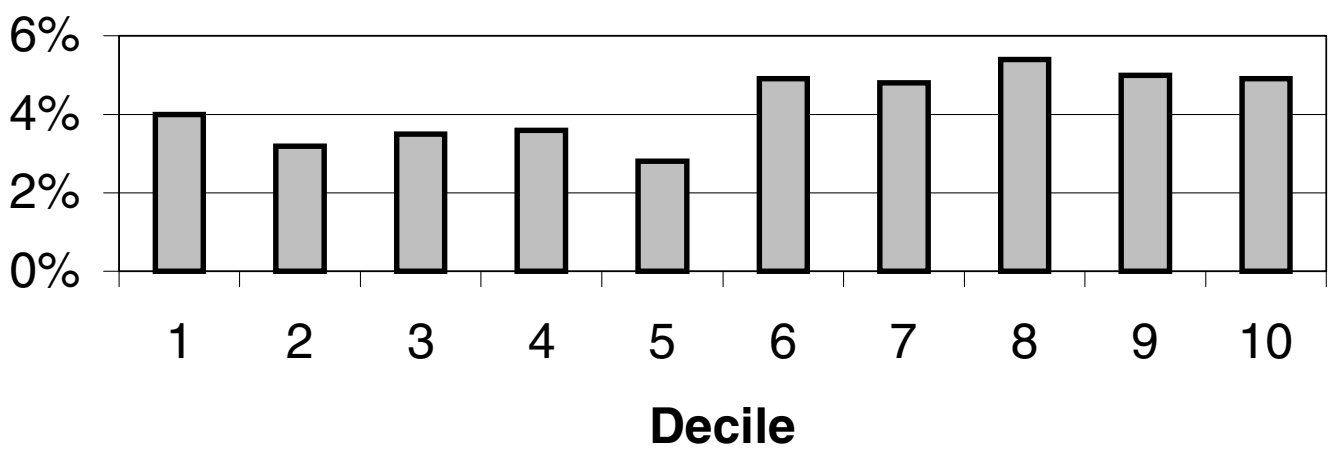

Figure 9 Response rates

new buyers. Simply, a more 'loyal' customer can be expected to respond in a superior manner.

Management reasoned that the aggressive marketing they had undertaken to acquire new customers was the primary cause of the results. They felt pleased about the company's efforts, after all, advertising, coupled with the data mining result, looked fairly impressive. Their conclusion was used to develop a set of rules for a subsequent mailing. The primary rule was to "Select "new" customers'. Six weeks later, the decile performance report in Figure 9 was generated.

What had happened? Why were response rates so poor in the lower deciles (the supposedly better segment being the newest customers), and so much better in the higher segments? What did the data mining software do? Upon further examination, it was discovered that the file used to develop the rules omitted a huge bloc of data. Indeed, 12 months of purchase history were deleted from this file. Only the multi-buyers possessed this past performance. With these data missing at the start, the data mining algorithms assumed from the analysis that first-time buyers performed better. This example demonstrates again the need for experienced eyes and brains. 


\section{DATA EXPLORATION}

The most critical part of a data mining exercise is data investigation, or exploration. Analysis is performed to eliminate data that provide no value to the analyst, as well as to select predictors that potentially provide benefit. Many data mining programs contain modules that perform these sorts of activities. The marketer who allows these program components to choose predictors automatically can, however, encounter major problems.

An example is an automobile manufacturer that seeks prospects that 'most' look like a current customer. Because the resulting model will be used on prospects, the firm realises it must use data that are available for this audience. Hence customer-specific data cannot be used.

External data were purchased, and would serve as the basis for variable selection for the model. Data arrived from the external data provider, and were appended to a sample of existing customers. This sample was compared to another group of potential prospects. The analyst chose a TREE-based tool to do his analysis on the two groups.

Essentially, TREE-based tools establish rules that are used to 'optimise' selection of prospects for subsequent contact. For example, a rule might look like this:

'Select prospects that simultaneously satisfy the following requirements:

- male gender

— income in excess of $\$ 40,000$

— resident of urban areas

- married

- owners of home in excess of $\$ 200,000$.'

Not only does the TREE algorithm detect the criteria, it then converts these criteria into program code that can be applied to a prospect list. This is, after all, what many data miners are looking for - a fully mechanical solution.

One criterion that the TREE identified had to do with the number of children in a particular household. The relationship that was ascertained is shown in Figure 10. With this analysis, the 'data mining software' concluded that households with nine children are more likely to own this manufacturer's vehicle. Indeed 15 per cent of these households own this particular car. Software code was automatically produced so that names could be selected. The model was used on 3.2 million names. The problem was that only 74 names met these criteria. The marketer was perplexed. How could this happen?

On investigation the analysts at the automobile firm found that when they had originally purchased the external data for analysis, the programmer responsible for assembling the files placed a ' 9 ' on those records where number of children was unknown. As they proceeded to select names, the vendor was using the data as they actually appeared on the data provider's files. The value of ' 9 ' actually meant nine; it did not mean missing. Only 74 names actually had nine children.

The lesson here is that no software should be used robotically. An analyst capable in both statistical tools and possessing specific industry knowledge (in this case, automobile domain competency) must look over the analysis in order to spot anomalies.

\section{POST-DATA MINING}

A data analyst for a book club marketer, has spent several weeks completing a data mining effort. The analyst is certain that the objectives of the project are consistent with the overall business goals of reducing attrition by 11 per cent. 

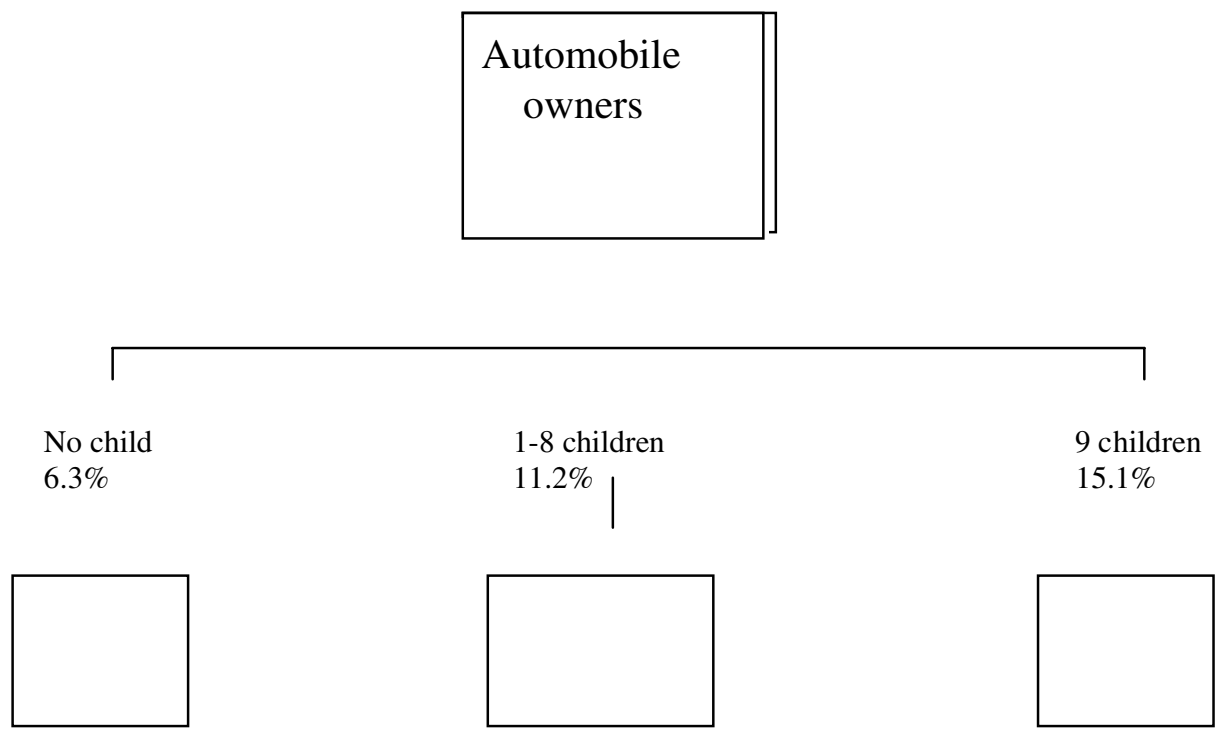

Figure 10 Ownership rates

Data preparation and exploration were meticulously managed. Results are logical and in line with expectations.

Management has decided to use the model results in conjunction with the company's new creative offer. Model code is sent to the firm that will be scoring and mailing. The marketer informs the vendor that 367,000 names will be evaluated with the model. Those in the top deciles are to receive a generous airline discount, if these customers renew their annual membership of the book club. The list is scored. The mailing takes place. The marketer, in order to gauge overall programme results, uses a control group. This segment will then be compared to the mail group to evaluate performance. Four months later, attrition rates are tabulated by decile for the control group. The results are displayed in Figure 11.

Something is wrong. How could attrition rates be lower in the top deciles for the control group? After all, by definition, no stimulus is presented to this segment. These might be good marketers, but impacting attrition rates for the control group in this manner did not seem logical.

The answer lies not in the strategic approach used by the marketer, but rather in the tactical implementation of the program. The vendor that scored the list assumed that higher model scores were associated with lower attrition, and lower scores indicated increased attrition. Based on the vendor's past experiences with response models, a higher score meant something good - a response. So here, too, a high score should mean something positive, as well, lower attrition.

Essentially, the vendor had sorted and thus deciled the file upside down.

While this may seem to be the most uncomplicated part of the data mining exercise, it can be the most devastating if inappropriately applied.

\section{CONCLUSION}

At a convention of statisticians, a survey was presented that concluded that most of the professionals that have been involved in data mining-related problems had already been utilising these analysis 


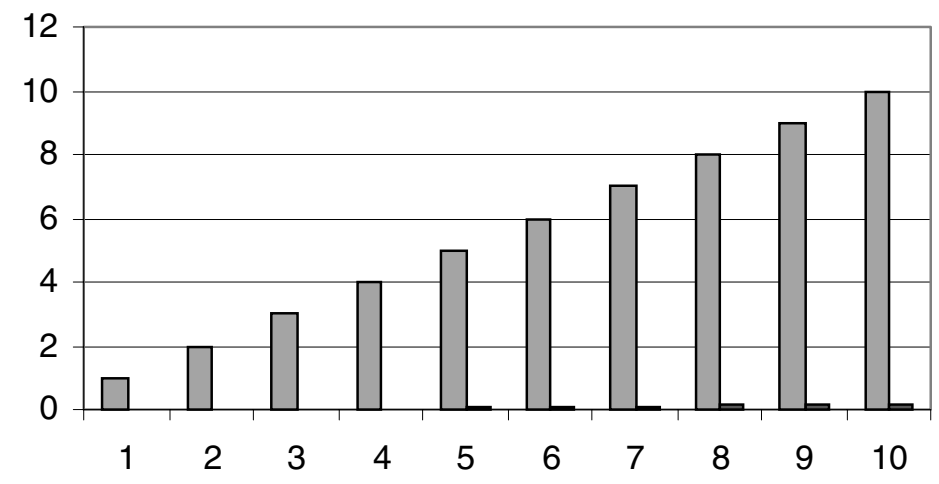

Figure 11 Attrition rates

techniques for the better part of the 1990s.

User-friendly data mining software, also introduced last decade, is hardly unwelcome. On the contrary, it has made the analytic experience more enjoyable by bringing together a variety of technologies under one roof. There are, however, ingredients in the overall scheme of data mining projects that this 'new' software does not, and perhaps cannot, address.

With the fairly universal agreement that the data mining analysis function of the software contributes only a small part of the overall project, marketers must be vigilant in ensuring that the final result is meaningful and useful. A clear business objective, understanding the data, dealing with oddities, and having clear quality control procedures are still essential ingredients in managing an analytic project.

Above all, a data miner with domain knowledge of the industry, and the ability to question and to challenge will help minimise the unexpected surprises that almost always materialise. 